\title{
Osteogenic Malignancy and Severe Vitamin D Deficiency (Oncogenic Osteomalacia)
}

Carol Postlethwaite', Amy Thomas², Thomas Ulahannan ${ }^{3}$, Jesse Kumar ${ }^{4}$

${ }^{1}$ StR Acute Medicine, ${ }^{2}$ Dietician, ${ }^{3}$ Locum Consultant Endocrinologist, ${ }^{4}$ Consultant Endocrinologist, Maidstone and Tunbridge Wells NHS Trust

\section{Introduction}

Vitamin D deficiency is increasingly being recognized as a metabolic disorder in temperate climates with various bone, cardiovascular and systemic manifestations. However topical, it is important to exclude tumour induced osteogenic osteomalacia as a possible aetiology in severe Vitamin D deficiency when other risk factors (vegetarian diet, ethnicity etc.) are absent. We would like to introduce and discuss the importance of FGF-23 (Fibroblast Growth Factor-23) as a tumor marker and paraneoplastic substance.

\section{Case Report}

A 58-year-old Caucasian man presented to his general practitioner with over one year of severe back pain and was discovered to have very low vitamin D3 levels $(<10 \mathrm{nmol} / \mathrm{L})$. Alkaline phosphatase was elevated at $160 \mathrm{U} / \mathrm{L}$ but calcium, phosphate and parathormone (PTH) levels at that time were normal. He was not a vegetarian and had normal exposure to sunlight. He had no constitutional symptoms or signs of thyroid disease, nor exposure to heavy metals or steroids. Over the next few months his calcium levels decreased to a low of 2.06 $\mathrm{mmol} / \mathrm{L}$ and phosphate to $0.76 \mathrm{mmol} / \mathrm{L}$. He was prescribed Vitamin D supplements and levels began to normalise. He developed increasing pain in his right femur; an HDP Tc-99m bone scan revealed marked increased activity in the right proximal femur indicating an aggressive osteoblastic process, in-keeping with an osteosarcoma. CT and MRI scans confimed a large tumour emanating from the right femoral head. Examination of a plain X-ray of the pelvis taken 6 months previous to this presentation showed changes consistent with the tumour, however the scan had not been reported by a radiologist. The femur was entirely resected at the Royal Orthopedic Hospital and a titanium implant inserted to allow limb salvage. Subsequent histology of the tumor revealed a type IIb chondrosarcoma. Repeated bone scan and interval CT scan revealed no local recurrence at 12 months. However, FGF-23 levels taken 14 months post surgery had not returned to the normal range and the patient remained biochemically Vitamin D deficient suggesting further ectopic areas of tumour growth.

\section{Radiology}

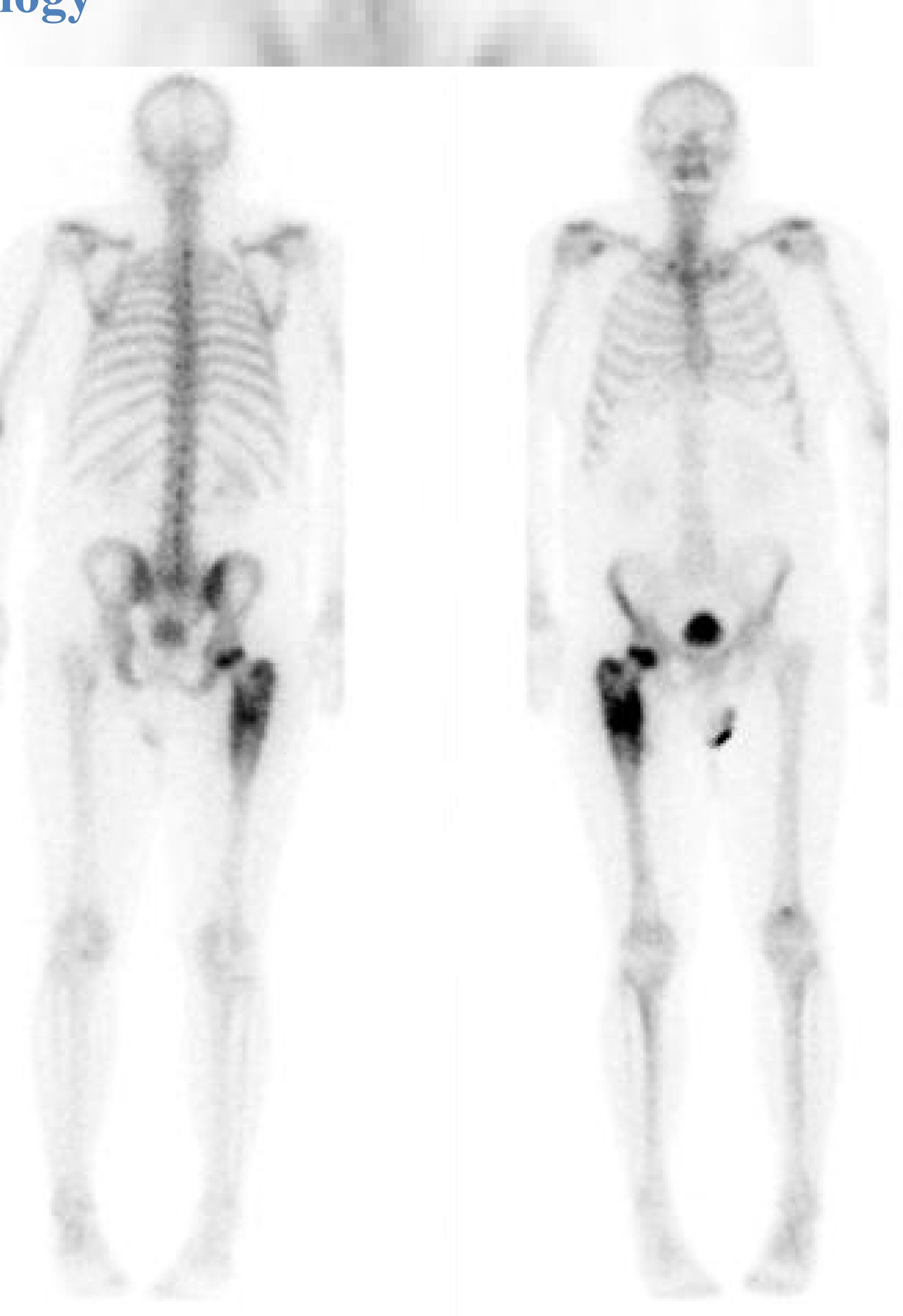

Figure 1. HDP Tc ${ }^{99 m}$ Bone Scintography.

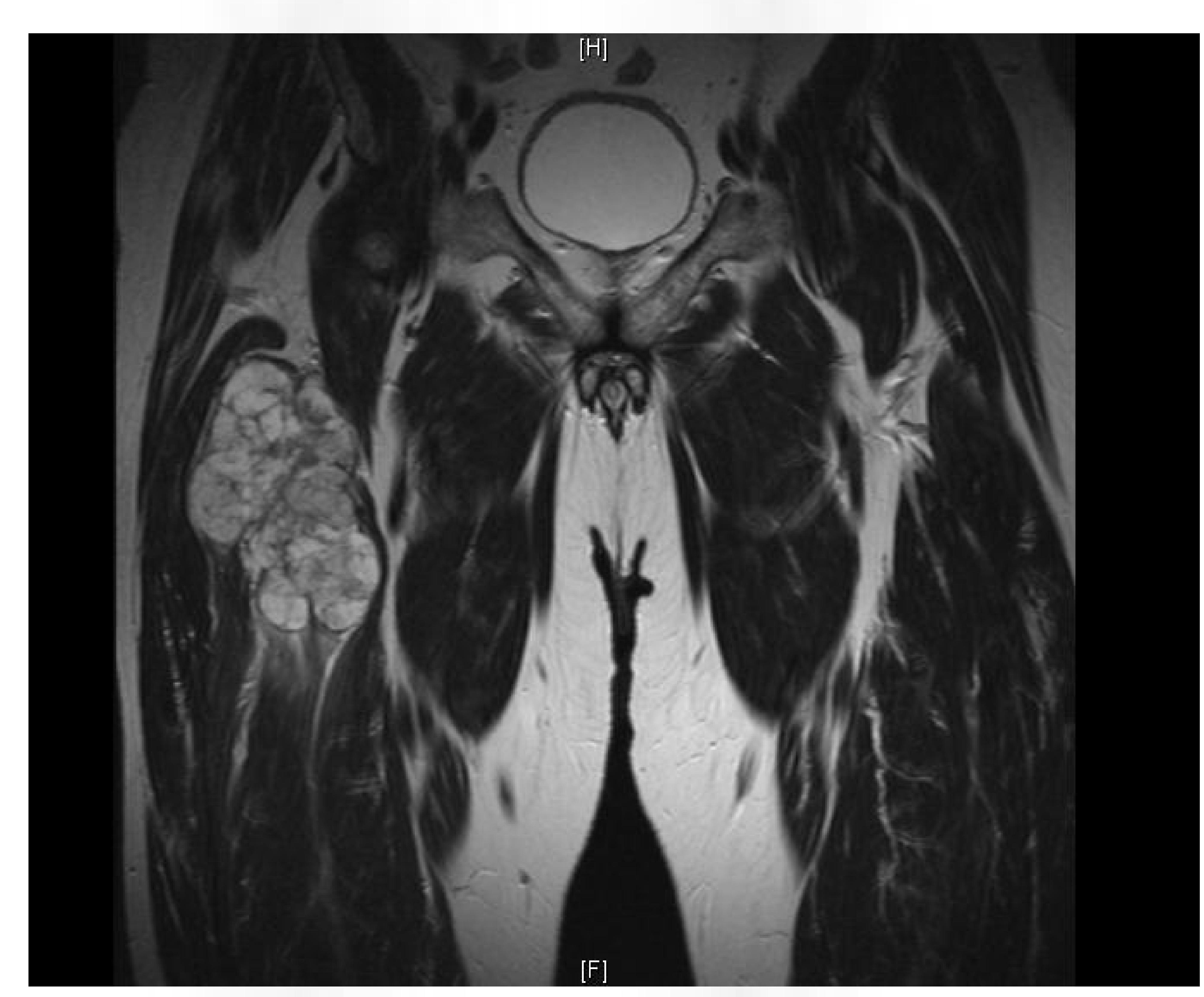

Figure 2. MRI anterior thigh

\section{Discussion}

Oncogenic Osteomalacia, also known as Tumour-induced Osteomalacia (TIO) is a rare paraneoplastic syndrome of abnormal phosphate and Vitamin D metabolism caused by typically small endocrine tumours that secrete FGF-23 a phosphatonin. Typical features include hypophosphataemia due to renal phosphate wasting, inappropriately low to normal Vitamin D levels and normal to elevated FGF-23. Patients suffer years of symptoms before diagnosis leading to bone pain, fractures, depression and muscle weakness. In our patient, the tumour was unusually large and so easily located after external signs of the tumour were present, however in many cases reported in the literature the tumour is never found Locating the typically benign tumour is paramount as TIO con be entirely cured if removed, provided it is not of a subtype that metastasises. There are four main classifications of TIO of which our patient had the most common variant: phosphaturic mesenchymal tumor (mixed connective tissue) PMTM$\mathrm{CT}$; a chondrosarcoma. Osteoblastoma-like variant, non-ossifying fibroma-like variant and ossifying fibroma-like variant complete the set. FGF-23 is highly expressed in the abnormal proliferating cells, and a positive immunostain for vimentin is also commonly found.

FGF-23 is a member of the FGF ligand superfamily and acts as an endocrine factor. Mutations in the genes controlling its expression have been found in autosomal dominant and recessive hypophosphataemic rickets, $\mathrm{X}$ linked hypophosphataemia and polyosteotic fibrous dysplasia. It is produced in the highest quantites in bone and lovels are regulated in reltion to serum phosphorus and 125 Vitamin $D$ levels. It binds to FGF receptors on number of tissues together with a cofactor - Klotho which is expressed and secreted in the distal nephron. It requires PTH to effect reduced Na-Pi $2 \mathrm{a}$ and c expression in the nephron causing phosphaturia. FGF-23 itself regulates 1,25 Vitamin D both downregulating 1 alpha hydroxylase and upregulating 24 hydroxylase reducing the concentration of 1,25 Vitamin D. High levels of FGF-23 secreted by tumours result in phosphaturia and low levels of Vitamin D causing a relatively resistent (to usual doses of supplements) osteomalacia. Confirmation of inappropriately normal or high FGF-23 in combination with low phosphate can exclude other diagnoses such as renal related Fanconi tubulopathies, burns, heavy metal exposure, cytotoxic drugs as well as haematological malignancies, refeeding syndrome or simple dietary deficiency.

Imaging of the tumour to guide surgery is the priority, however as they are often small this can be challenging. Tc ${ }^{99 m}$ HDP bone scans are too sensitive and not specific, also picking up active arthritis and recent fractures as can be seen on figure 1. More useful is positron emission tomography linked with CT (FDG PET-CT) which is available in most cancer centres in the UK. It is also not specific identifying high metabolic areas. Indium ${ }^{111}$ and Octreotide labelled scans have shown promise. All scans should cover the whole skeleton, not missing the hands or feet. MRI can then confirm location of the tumour. Post-op there should be a rapid drop in the level of FGF-23 (hours) and normalisation of the phosphate levels within 2 days. Skeletal remineralisation can take up to one year. Whilst metastases are rare, the lung is the most common site. FGF-23 can be used to supplement routine radiology follow-up. There is no role for cytotoxic chemotherapy or radiotherapy, however radiofrequency ablation has shown promise.

In patients when surgery is not possible pharmacological manipulation of the hypophosphataemia is possible using phosphate supplements and alfacalcidol / calcitriol or cinacalcet can be used to treat the secondary hyperparathyroidism. Problems include gastric upset secondary to the phosphate supplements and hypercalciuria with nephrolithiasis which can be ameliorated with thiazides.

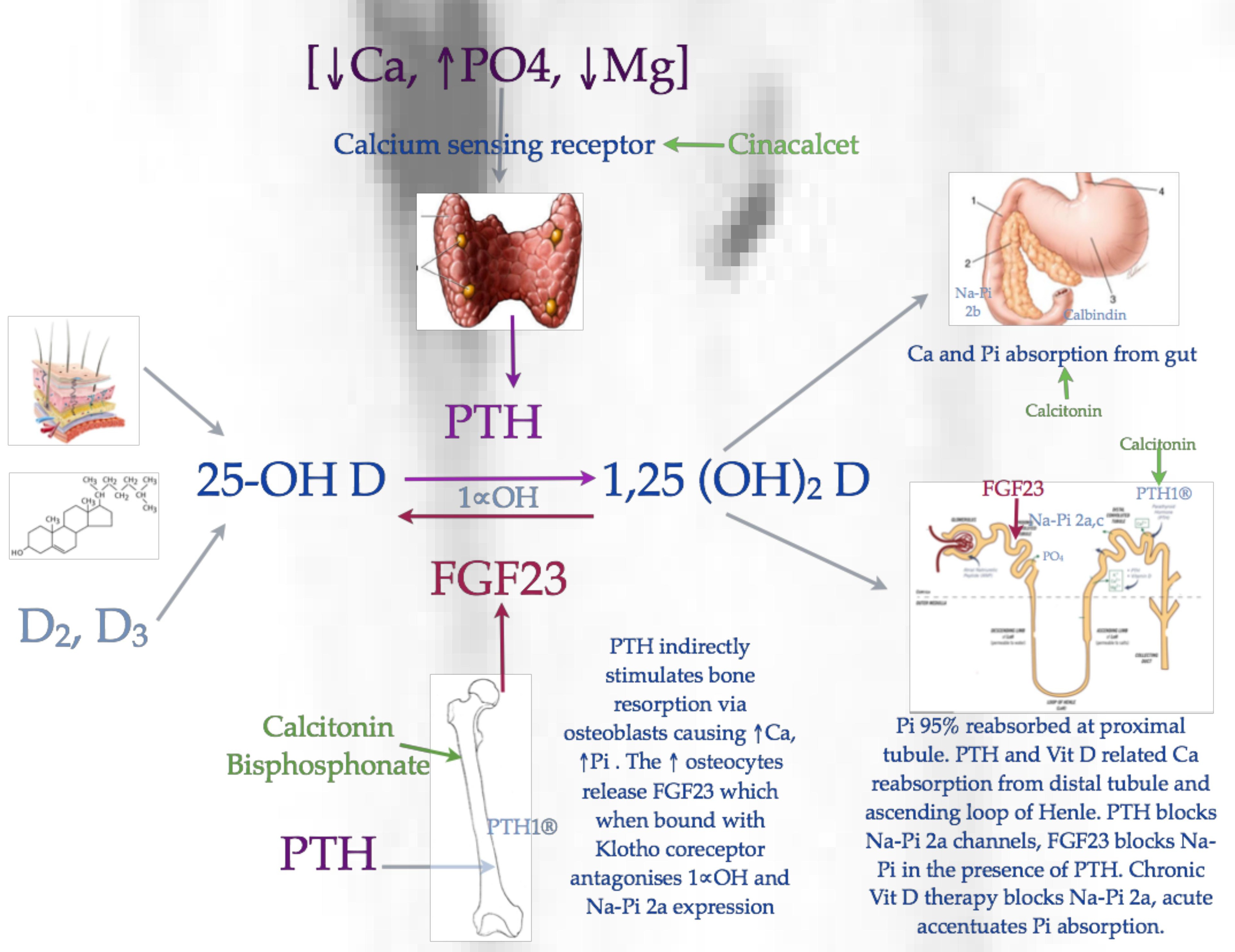

Conclusion

Osteogenic osteomalacia is a very rare diagnosis with subtle manifestations which are considered a consequence of an underlying neoplasm, which can be very indolent. Undetected, the associated malignant tumors could metastasize. A high degree of clinical awareness and suspicion is required, particularly if the tumor is small along with the use of radiological investigations such as PET-CT and Octreotide scans to detect occult tumours. FGF-23 is a member of the FGF family which is involved in phosphate homeostasis and skeletogenesis and may prove useful as a tumour marker to help exclude a malignant tumour in severe Vitamin D deficiency.

\section{References}

FIRENZA, F, ABUDU, A, RJ GRIMER, SR CARTER, TILLMAN, R, AYOUB, K, MANGHAM, D. \& DAVIES, A. 2002. Risk
factors for survival and local control in chondrosarcoma of bone., J Bone Joint Surg (Br), 84-B.

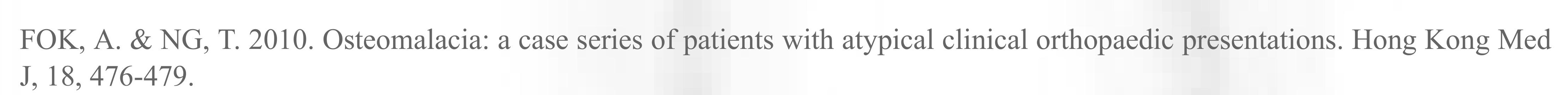

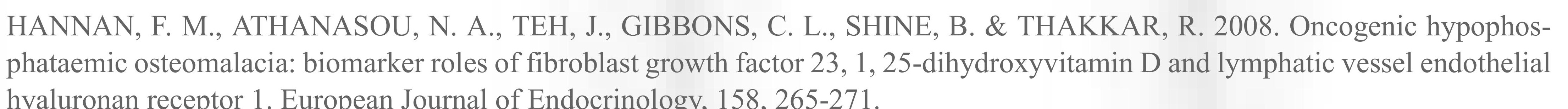

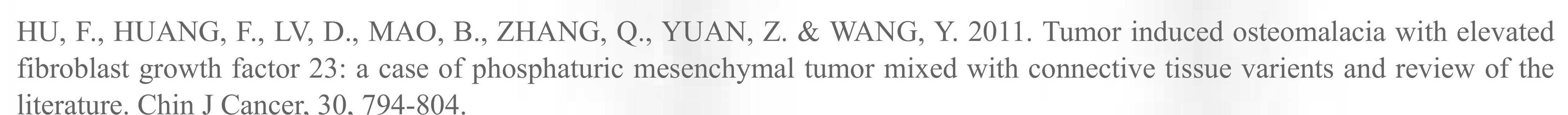
JL GELLER, KHOSRAVI, A., KELLY, M. RIMINUCCI, M. ADAMS, J. \& COLLINS, M. 2007. Cinacalcet in the management
of tumor induced osteomalacia.J Bone Miner Res, 22,931-7.

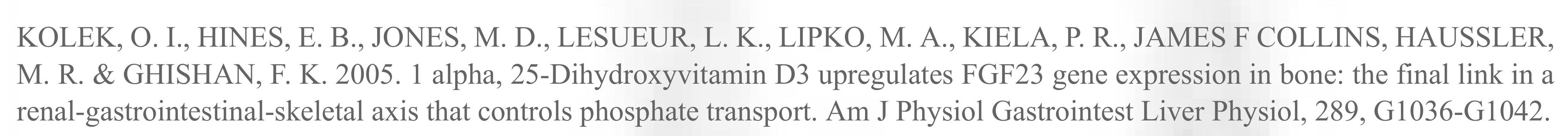
HANMUGA SUNDARAM PALANISWAMY, PADMA SUBRAMANYAM \& KUMAR, H. 2011. Oncogenic Osteomalacia diagnosed by pool scintography. Indian Journal of Nuclear Medicine, 26, 188-191. WILLIAM H CHONG, A. M., CLARA C CHEN, MICHAEL T COLLINS 2011. Tumor induced Osteomalacia. Endocrine-Related
Cancer, 18 , R33-R77.

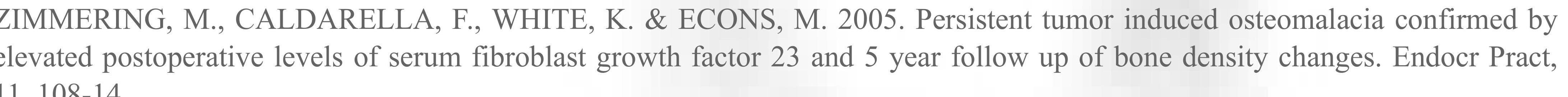

